\title{
A staggered scheme for the Euler equations.
}

Thierry Goudon, Julie Llobell and Sebastian Minjeaud

\begin{abstract}
We extend to the full Euler system the scheme introduced in [Berthelin, Goudon, Minjeaud, Math. Comp. 2014] for solving the barotropic Euler equations. This finite volume scheme is defined on staggered grids with numerical fluxes derived in the spirit of kinetic schemes. The difficulty consists in finding a suitable treatment of the energy equation while density and internal energy on the one hand, and velocity on the other hand, are naturally defined on dual locations. The proposed scheme uses the density, the velocity and the internal energy as computational variables and stability conditions are identified in order to preserve the positivity of the discrete density and internal energy. Moreover, we define averaged energies which satisfy local conservation equations. Finally, we provide numerical simulations of Riemann problems to illustrate the behaviour of the scheme.
\end{abstract}

Key words: Finite Volumes, Conservation laws, Staggered grids, Euler equations

MSC (2010): 65M08, 76M12, 35L65, 35Q31

\section{Introduction.}

This work aims at designing a scheme to numerically solve the 1D-Euler system:

$$
\begin{aligned}
& \partial_{t}\left(\begin{array}{c}
\rho \\
\rho u \\
\rho E
\end{array}\right)+\partial_{x}\left(\begin{array}{c}
\rho u \\
\rho u^{2}+p \\
\rho E u+p u
\end{array}\right)=0, \quad(t, x) \in[0, \infty) \times \mathbb{R}, \\
& E=u^{2} / 2+e, \quad p=(\gamma-1) \rho e,
\end{aligned}
$$

Thierry Goudon, e-mail: thierry.goudon@inria.fr Julie Llobell, e-mail: 1lobell@unice.fr

Sebastian Minjeaud, e-mail: sebastian.minjeaud@unice.fr

Université Côte d'Azur, CNRS, Inria, LJAD, France 
where $\rho, u, E$ and $p$ stand for the density, the velocity, the total energy and the pressure respectively, $u^{2} / 2$ and $e$ are the kinetic and internal energies, and $\gamma>1$ is the adiabatic exponent.

We wish to extend to (1) the scheme designed in [1] for the barotropic Euler equations. This scheme works on staggered grids - meaning that densities and velocities are not collocated - and this raises a difficulty for (1) as the definition of the total energy mixes quantities, namely the velocity and the internal energy, naturally defined on different grids. To address this issue, it is convenient to work with the internal energy equation, namely

$$
\partial_{t}(\rho e)+\partial_{x}(\rho e u)=-p \partial_{x} u,
$$

instead of the evolution equation for $\rho E$, since discrete densities, pressures, and internal energies are naturally stored at the same locations. This formulation has also the advantage of making more direct the analysis of the positivity of $e$. Unfortunately, as it is well-known, this non conservative formulation is not equivalent to (1) when the solution presents discontinuities. We shall follow the approach discussed in [2] by introducing in (2) correction terms accounting for the kinetic energy balance. Then, the scheme introduced in [2] can be shown: a) to be consistent with (a weak form of) the total energy equation as the space step $\delta x$ goes to zero and b) to conserve the global discrete total energy. Our purpose is two-fold. First of all, we shall adapt the scheme of [1] for dealing with (1). Second of all, we introduce averaged energies which satisfy local conservation equations. Finally we provide some numerical simulations in Section 5.

\section{Staggered scheme.}

Let $\left(x_{j}\right)_{j}$ be a subdivision of the 1D computational domain and denote the size of the cells by $\delta x_{j+\frac{1}{2}}=x_{j+1}-x_{j}$. The cell centers, $x_{j+\frac{1}{2}}=\left(x_{j}+x_{j+1}\right) / 2$, define the dual mesh and we set $\delta x_{j}=\left(\delta x_{j-\frac{1}{2}}+\delta x_{j+\frac{1}{2}}\right) / 2$. The discrete densities $\rho_{j+\frac{1}{2}}$ and internal energies $e_{j+\frac{1}{2}}$ are stored at the centers $x_{j+\frac{1}{2}}$ whereas the velocities $u_{j}$ are located at the edges $x_{j}$. The time dicretization is explicit and we use the convention that, with $q$ the evaluation of a certain quantity at time $t, \bar{q}$ stands for its update at time $t+\delta t$.

- The density $\rho_{j+\frac{1}{2}}$ is updated using the discrete mass balance equation:

$$
\frac{\bar{\rho}_{j+\frac{1}{2}}-\rho_{j+\frac{1}{2}}}{\delta t}+\frac{\mathscr{F}_{j+1}-\mathscr{F}_{j}}{\delta x_{j+\frac{1}{2}}}=0 .
$$

The mass fluxes are defined by $\mathscr{F}_{j}=\mathscr{F}_{j}^{+}+\mathscr{F}_{j}^{-}$where $\mathscr{F}_{j}^{+}=\mathscr{F}^{+}\left(\rho_{j-\frac{1}{2}}, e_{j}, u_{j}\right)$, $\mathscr{F}_{j}^{-}=\mathscr{F}^{-}\left(\rho_{j+\frac{1}{2}}, e_{j}, u_{j}\right)$ and $e_{j}=\left(e_{j-\frac{1}{2}}+e_{j+\frac{1}{2}}\right) / 2$. Denoting $c(e)=\sqrt{(\gamma-1) \gamma e}$ 
the sound speed, the definition of the numerical fluxes $\mathscr{F}^{ \pm}$is extracted from [1]

$$
\mathscr{F}^{+}(\rho, e, u)= \begin{cases}0 & \text { if } u \leqslant-c(e), \\ \frac{\rho(u+c(e))^{2}}{4 c(e)} & \text { if }|u|<c(e), \text { and } \mathscr{F}^{-}(\rho, e, u)=-\mathscr{F}^{+}(\rho, e,-u) . \\ \rho u & \text { if } u>c(e),\end{cases}
$$

In the sequel we use the following two properties: $\forall u \in \mathbb{R}, \forall \rho, e \geqslant 0$,

$$
0 \leqslant \mathscr{F}^{+}(\rho, e, u) \leqslant \rho\left[\lambda_{+}(e, u)\right]^{+} \quad \text { and } \quad-\rho\left[\lambda_{-}(e, u)\right]^{-} \leqslant \mathscr{F}^{-}(\rho, e, u) \leqslant 0,
$$

where $\lambda_{ \pm}(e, u)=u \pm c(e)$ and $[z]^{ \pm}=\frac{1}{2}(|z| \pm z)$.

- The velocity $u_{j}$ is then updated using the discrete momentum balance equation:

$$
\frac{\bar{\rho}_{j} \bar{u}_{j}-\rho_{j} u_{j}}{\delta t}+\frac{\mathscr{G}_{j+\frac{1}{2}}-\mathscr{G}_{j-\frac{1}{2}}}{\delta x_{j}}+\frac{\Pi_{j+\frac{1}{2}}-\Pi_{j-\frac{1}{2}}}{\delta x_{j}}=0 .
$$

The momentum flux $\mathscr{G}_{j+\frac{1}{2}}$ and the pressure $\Pi_{j+\frac{1}{2}}$ are defined by:

$$
\mathscr{G}_{j+\frac{1}{2}}=u_{j} \mathscr{F}_{j+\frac{1}{2}}^{+}+u_{j+1} \mathscr{F}_{j+\frac{1}{2}}^{-} \quad \text { and } \quad \Pi_{j+\frac{1}{2}}=(\gamma-1) \rho_{j+\frac{1}{2}} e_{j+\frac{1}{2}} .
$$

The quantities $\rho_{j}$ and $\mathscr{F}_{j+\frac{1}{2}}^{ \pm}$are expressed as mean values of $\rho_{j \pm \frac{1}{2}}$ and $\mathscr{F}_{j}^{ \pm}, \mathscr{F}_{j+1}^{ \pm}$:

$$
\rho_{j}=\frac{\delta x_{j+\frac{1}{2}} \rho_{j+\frac{1}{2}}+\delta x_{j-\frac{1}{2}} \rho_{j-\frac{1}{2}}}{2 \delta x_{j}} \quad \text { and } \quad \mathscr{F}_{j+\frac{1}{2}}^{ \pm}=\frac{\mathscr{F}_{j+1}^{ \pm}+\mathscr{F}_{j}^{ \pm}}{2}
$$

- The internal energy $e_{j+\frac{1}{2}}$ is updated using the following discrete equation:

$$
\frac{\bar{\rho}_{j+\frac{1}{2}} \bar{e}_{j+\frac{1}{2}}-\rho_{j+\frac{1}{2}} e_{j+\frac{1}{2}}}{\delta t}+\frac{\mathscr{E}_{j+1}-\mathscr{E}_{j}}{\delta x_{j+\frac{1}{2}}}+\Pi_{j+\frac{1}{2}} \frac{\bar{u}_{j+1}-\bar{u}_{j}}{\delta x_{j+\frac{1}{2}}}=S_{j+\frac{1}{2}} .
$$

The internal energy flux $\mathscr{E}_{j}$ is given by $\mathscr{E}_{j}=e_{j-\frac{1}{2}} \mathscr{F}_{j}^{+}+e_{j+\frac{1}{2}} \mathscr{F}_{j}^{-}$. According to [2], the rhs $S_{j+\frac{1}{2}}$ is designed to account for the rest term that appears in the discrete kinetic energy balance and that do not vanish when $\delta x$ goes to zero.

- To be more specific, the kinetic energy balance is obtained by multiplying (4) by $\bar{u}_{j}$. We find, see $[1,2]$ :

$$
\frac{\bar{\rho}_{j} \frac{\bar{u}_{j}^{2}}{2}-\rho_{j} \frac{u_{j}^{2}}{2}}{\delta t}+\frac{\mathscr{H}_{j+\frac{1}{2}}-\mathscr{H}_{j-\frac{1}{2}}}{\delta x_{j}}+\frac{\Pi_{j+\frac{1}{2}}-\Pi_{j-\frac{1}{2}}}{\delta x_{j}} \bar{u}_{j}=-R_{j},
$$

where the kinetic energy flux is given by $\mathscr{H}_{j+\frac{1}{2}}=\frac{u_{j}^{2}}{2} \mathscr{F}_{j+\frac{1}{2}}^{+}+\frac{u_{j+1}^{2}}{2} \mathscr{F}_{j+\frac{1}{2}}^{-}$and 


$$
\begin{aligned}
R_{j} & =\frac{1}{2 \delta t} \bar{\rho}_{j}\left(\bar{u}_{j}-u_{j}\right)^{2}+\frac{1}{\delta x_{j}}\left(\frac{\left(u_{j}-u_{j-1}\right)^{2}}{2} \mathscr{F}_{j-\frac{1}{2}}^{+}-\frac{\left(u_{j+1}-u_{j}\right)^{2}}{2} \mathscr{F}_{j+\frac{1}{2}}^{-}\right) \\
& +\frac{1}{\delta x_{j}}\left(\bar{u}_{j}-u_{j}\right)\left(u_{j}-u_{j-1}\right) \mathscr{F}_{j-\frac{1}{2}}^{+}+\frac{1}{\delta x_{j}}\left(\bar{u}_{j}-u_{j}\right)\left(u_{j+1}-u_{j}\right) \mathscr{F}_{j+\frac{1}{2}}^{-}
\end{aligned}
$$

It is thus quite natural to define the source term in the following way:

$$
S_{j+\frac{1}{2}}=\frac{\delta x_{j+1} R_{j+1}+\delta x_{j} R_{j}}{2 \delta x_{j+\frac{1}{2}}}
$$

The scheme presented above is close to the $1 \mathrm{D}$ version of the scheme presented in [3] but the two schemes differ by two points. Firstly, the mass fluxes in [3] are upwinded with respect to the material velocity (in other words, it corresponds to the choice $\left.\mathscr{F}^{ \pm}(\rho, e, u)= \pm \rho[u]^{ \pm}\right)$. Secondly, the time steppings are different: even if both schemes are explicit, the variables are not updated in the same order. We solve the discrete equations in the following way: $\rho \rightarrow u \rightarrow e$ whereas [3] proceeds with $\rho \rightarrow e \rightarrow u$. In particular, here the corrective term $S_{j+\frac{1}{2}}$ does not need any time shift since the updated velocity $\bar{u}$ is known when solving (6).

\section{Stability conditions.}

We now turn to the study of the stability conditions which ensure the positivity of the density and the internal energy.

Proposition 1. Assuming that $e_{j+\frac{1}{2}} \geqslant 0, \rho_{j+\frac{1}{2}} \geqslant 0, \forall j$ and the following CFL-like conditions hold for all $j$

$$
\begin{gathered}
\frac{\delta t}{\delta x_{j+\frac{1}{2}}}\left(\left[u_{j+1}\right]^{+}+\frac{c\left(e_{j+\frac{3}{2}}\right)+c\left(e_{j+\frac{1}{2}}\right)}{\sqrt{2}}+\left[u_{j}\right]^{-}+\frac{c\left(e_{j+\frac{1}{2}}\right)+c\left(e_{j-\frac{1}{2}}\right)}{\sqrt{2}}\right) \leqslant \frac{1}{\gamma}, \\
\frac{\delta t}{\delta x_{j+\frac{1}{2}}} c\left(e_{j+\frac{1}{2}+k}\right) \leqslant \frac{(\gamma-1)}{2 \sqrt{2}}, \quad \forall k \in\{-1,0,1\}, \\
\text { then } \bar{e}_{j+\frac{1}{2}} \geqslant 0 \text { and } \bar{\rho}_{j+\frac{1}{2}} \geqslant 0 \text {. }
\end{gathered}
$$

Proof. We assume that $e_{j+\frac{1}{2}} \geqslant 0, \rho_{j+\frac{1}{2}} \geqslant 0$ and that (7) and (8) holds for all $j$. We start by observing that:

$$
\begin{gathered}
{\left[\lambda_{ \pm}(e, u)\right]^{ \pm} \leqslant[u]^{ \pm}+c(e)} \\
\sqrt{2} c\left(e_{j}\right) \leqslant c\left(e_{j-\frac{1}{2}}\right)+c\left(e_{j+\frac{1}{2}}\right)
\end{gathered}
$$

Positivity of the density: As proved in [1], the positivity of $\bar{\rho}_{j+\frac{1}{2}}$ comes from the inequality 


$$
\frac{\delta t}{\delta x_{j+\frac{1}{2}}}\left(\left[\lambda_{+}\left(e_{j+1}, u_{j+1}\right)\right]^{+}+\left[\lambda_{-}\left(e_{j}, u_{j}\right)\right]^{-}\right) \leqslant 1 .
$$

It is directly implied by (7) since $\gamma>1$ and (9) holds.

Positivity of the internal energy: We rewrite the terms $(-1)^{i} \Pi_{j+\frac{1}{2}} \bar{u}_{j+i}, i \in\{0,1\}$, involved in (6), by making the discrete time derivative $\left(\bar{u}_{j+i}-u_{j+i}\right)$ appear. Then, we make use of the Young inequality as follows:

$$
\begin{aligned}
& (-1)^{i} \Pi_{j+\frac{1}{2}} \bar{u}_{j+i}=(-1)^{i}(\gamma-1)\left(\rho_{j+\frac{1}{2}} e_{j+\frac{1}{2}}\left(\bar{u}_{j+i}-u_{j+i}\right)+\rho_{j+\frac{1}{2}} e_{j+\frac{1}{2}} u_{j+i}\right) \\
& \geqslant-\rho_{j+\frac{1}{2}}\left(\frac{c\left(e_{j+\frac{1}{2}}\right)}{2 \sqrt{2} \gamma}\left(\bar{u}_{j+i}-u_{j+i}\right)^{2}+(\gamma-1) e_{j+\frac{1}{2}}\left(\frac{c\left(e_{j+\frac{1}{2}}\right)}{\sqrt{2}}-(-1)^{i} u_{j+i}\right)\right) .
\end{aligned}
$$

Next, we write $\bar{\rho}_{j+\frac{1}{2}} \bar{e}_{j+\frac{1}{2}} \geqslant \mathrm{~T}_{0}+\mathrm{T}_{1}^{0}+\mathrm{T}_{1}^{1}$ where:

$$
\begin{aligned}
& \mathrm{T}_{0}=\rho_{j+\frac{1}{2}} e_{j+\frac{1}{2}}\left(1-\frac{\delta t}{\delta x_{j+\frac{1}{2}}}(\gamma-1)\left(2 \frac{c\left(e_{j+\frac{1}{2}}\right)}{\sqrt{2}}-u_{j}+u_{j+1}\right)\right)-\delta t \frac{\mathscr{E}_{j+1}-\mathscr{E}_{j}}{\delta x_{j+\frac{1}{2}}} \\
& \mathrm{~T}_{1}^{i}=\frac{\delta t}{2} \frac{\delta x_{j+i}}{\delta x_{j+\frac{1}{2}}} R_{j+i}-\frac{\delta t}{\delta x_{j+\frac{1}{2}}} \frac{c\left(e_{j+\frac{1}{2}}\right)}{2 \sqrt{2} \gamma} \rho_{j+\frac{1}{2}}\left(\bar{u}_{j+i}-u_{j+i}\right)^{2} .
\end{aligned}
$$

Thus, to guarantee that $\bar{e}_{j+\frac{1}{2}}$ is non negative it is sufficient to ensure that these three terms are non negative. This holds under the assumptions (7) and (8).

Indeed, using the definition of the flux $\mathscr{E}_{j}$ and owing to (3), we obtain

$$
\begin{aligned}
\mathrm{T}_{0} \geqslant \rho_{j+\frac{1}{2}} e_{j+\frac{1}{2}} & \left(1-\frac{\delta t}{\delta x_{j+\frac{1}{2}}}(\gamma-1)\left(\left[u_{j}\right]^{-}+\frac{c\left(e_{j+\frac{1}{2}}\right)}{\sqrt{2}}+\left[u_{j+1}\right]^{+}+\frac{c\left(e_{j+\frac{1}{2}}\right)}{\sqrt{2}}\right)\right) \\
& -\frac{\delta t}{\delta x_{j+\frac{1}{2}}} \rho_{j+\frac{1}{2}} e_{j+\frac{1}{2}}\left(\left[\lambda_{+}\left(e_{j+1}, u_{j+1}\right)\right]^{+}+\left[\lambda_{-}\left(e_{j}, u_{j}\right)\right]^{-}\right)
\end{aligned}
$$

where, due to (7), the rhs is non negative by virtue of (9) and (10) .

Next, we turn to $T_{1}^{i}$. Using twice the Young inequality and bearing in mind the definition of $\bar{\rho}_{j}$, we observe that

$$
\frac{\delta t}{2} \frac{\delta x_{j+i}}{\delta x_{j+\frac{1}{2}}} R_{j+i} \geqslant \frac{\delta x_{j+i}}{4 \delta x_{j+\frac{1}{2}}}\left(\bar{u}_{j+i}-u_{j+i}\right)^{2}\left(\rho_{j+i}-\frac{\delta t}{\delta x_{j+i}}\left(\mathscr{F}_{j+i+\frac{1}{2}}^{+}-\mathscr{F}_{j+i-\frac{1}{2}}^{-}\right)\right) .
$$

Hence, we have

$$
\mathrm{T}_{1}^{i} \geqslant \frac{\delta x_{j+i}}{4 \delta x_{j+\frac{1}{2}}}\left(\bar{u}_{j+i}-u_{j+i}\right)^{2}\left(\rho_{j+i}-\frac{\delta t}{\delta x_{j+i}}\left(\mathscr{F}_{j+i+\frac{1}{2}}^{+}-\mathscr{F}_{j+i-\frac{1}{2}}^{-}\right)-\frac{\delta t}{\gamma} \frac{2}{\sqrt{2}} \frac{\rho_{j+\frac{1}{2}} c\left(e_{j+\frac{1}{2}}\right)}{\delta x_{j+i}}\right) .
$$


Coming back to (5), we write $T_{1}^{i} \geqslant \frac{\left(\bar{u}_{j+i}-u_{j+i}\right)^{2}}{4 \delta x_{j+\frac{1}{2}}}\left(T_{2}^{i, 0}+T_{2}^{i, 1}\right)$ where, for $k=0,1$,

$$
\mathrm{T}_{2}^{i, k}=\frac{\delta x_{j+i+k-\frac{1}{2}}}{2} \rho_{j+i+k-\frac{1}{2}}-\delta t \frac{\mathscr{F}_{j+i+k}^{+}-\mathscr{F}_{j+i+k-1}^{-}}{2}-\frac{\delta t}{\gamma} \frac{2}{\sqrt{2}} \rho_{j+i+k-\frac{1}{2}} c\left(e_{j+\frac{1}{2}}\right) .
$$

Note that a non negative term has been added to obtain a symmetric formulation in the above inequality. Due to (3) and (7) we get

$$
\mathscr{F}_{j+i+k}^{+}-\mathscr{F}_{j+i+k-1}^{-} \leqslant \frac{\delta x_{j+i+k-\frac{1}{2}}}{\gamma \delta t} \rho_{j+i+k-\frac{1}{2}},
$$

and this allows us to write

$$
\mathrm{T}_{2}^{i, k} \geqslant \frac{\delta x_{j+i+k-\frac{1}{2}}}{2 \gamma} \rho_{j+i+k-\frac{1}{2}}\left(\gamma-1-\frac{\delta t}{\delta x_{j+i+k-\frac{1}{2}}} \frac{4}{\sqrt{2}} c\left(e_{j+\frac{1}{2}}\right)\right) .
$$

We conclude by observing that this term is non negative by virtue of (8).

\section{Numerical diffusion and energy conservation.}

It is worth discussing the expression of the numerical diffusion produced by our scheme, see also the appendix in [1]. Let us set the following non negative quantity

$$
C_{j}= \begin{cases}-u_{j} & \text { if } u_{j} \leqslant-c\left(e_{j}\right) \\ \frac{u_{j}^{2}+c\left(e_{j}\right)^{2}}{4 c\left(e_{j}\right)} & \text { if }\left|u_{j}\right|<c\left(e_{j}\right) \\ u_{j} & \text { if } u_{j}>c\left(e_{j}\right)\end{cases}
$$

and the following notations for averaged quantities

$$
\{q\}_{j}=\frac{q_{j-\frac{1}{2}}+q_{j+\frac{1}{2}}}{2} \text { and }\{q\}_{j+\frac{1}{2}}=\frac{q_{j}+q_{j+1}}{2} .
$$

Denoting $\mathscr{F}|\cdot|=\mathscr{F}^{+}-\mathscr{F}^{-}$, wich is a positive quantity, the mass and the momentum fluxes can be cast as the sum of a centered term and a diffusion term:

$$
\begin{aligned}
\mathscr{F}_{j} & =\{\rho\}_{j} u_{j}-\frac{C_{j}}{2}\left(\rho_{j+\frac{1}{2}}-\rho_{j-\frac{1}{2}}\right), \\
\mathscr{G}_{j+\frac{1}{2}} & =\{\mathscr{F}\}_{j+\frac{1}{2}}\{u\}_{j+\frac{1}{2}}-\frac{\{\mathscr{F} \mid \cdot\}_{j+\frac{1}{2}}}{2}\left(u_{j+1}-u_{j}\right) .
\end{aligned}
$$

Concerning the internal energy and kinetic energy fluxes, they become: 


$$
\begin{aligned}
\mathscr{E}_{j} & =\{\rho e\}_{j} u_{j}-\frac{C_{j}}{2}\left(e_{j+\frac{1}{2}} \rho_{j+\frac{1}{2}}-e_{j-\frac{1}{2}} \rho_{j-\frac{1}{2}}\right), \\
\mathscr{H}_{j+\frac{1}{2}} & =\{\mathscr{F}\}_{j+\frac{1}{2}}\left\{\frac{u^{2}}{2}\right\}_{j+\frac{1}{2}}-\frac{\{\mathscr{F} \mid \cdot\}_{j+\frac{1}{2}}}{2}\left(\frac{u_{j+1}^{2}}{2}-\frac{u_{j}^{2}}{2}\right) .
\end{aligned}
$$

As a by-product, it is remarkable that the scheme properly deals with 1D-contact discontinuities: if the discrete velocity and pressure are constant in the neighborhood of $x_{j+\frac{1}{2}}$, ie $u_{j-1}=u_{j}=u_{j+1}=u_{j+2}=u$ and $\Pi_{j-1 / 2}=\Pi_{j+1 / 2}=\Pi_{j+3 / 2}=\Pi$, then the scheme guaranties that they remain constant in the neighborhood of this point at the next time, ie $\bar{\Pi}_{j+1 / 2}=\Pi$ and $\bar{u}_{j+1}=u=\bar{u}_{j}$.

Let us now introduce the averaged energy in $x_{j+\frac{1}{2}}$ and $x_{j}$ defined by:

$$
E_{j+\frac{1}{2}}=e_{j+\frac{1}{2}}+\frac{\delta x_{j} \rho_{j} \frac{u_{j}^{2}}{2}+\delta x_{j+1} \rho_{j+1} \frac{u_{j+1}^{2}}{2}}{2 \delta x_{j+\frac{1}{2}} \rho_{j+\frac{1}{2}}}
$$

and

$$
E_{j}=\frac{u_{j}^{2}}{2}+\frac{\delta x_{j+\frac{1}{2}} \rho_{j+\frac{1}{2}} e_{j+\frac{1}{2}}+\delta x_{j-\frac{1}{2}} \rho_{j-\frac{1}{2}} e_{j-\frac{1}{2}}}{2 \delta x_{j} \rho_{j}} .
$$

To obtain conservative equations for those quantities, we introduce the fluxes

$$
\mathscr{T}_{j}=\mathscr{E}_{j}+\frac{\mathscr{H}_{j+\frac{1}{2}}+\mathscr{H}_{j-\frac{1}{2}}}{2} \text { and } \mathscr{T}_{j+\frac{1}{2}}^{*}=\frac{\mathscr{E}_{j+1}+\mathscr{E}_{j}}{2}+\mathscr{H}_{j+\frac{1}{2}}-\frac{\delta x_{j+1} R_{j+1}-\delta x_{j} R_{j}}{4}
$$

Next we get the following consistent balance equations for $\rho_{j} E_{j}$ and $\rho_{j+\frac{1}{2}} E_{j+\frac{1}{2}}$ :

$$
\frac{\bar{\rho}_{j+\frac{1}{2}} \bar{E}_{j+\frac{1}{2}}-\rho_{j+\frac{1}{2}} E_{j+\frac{1}{2}}}{\delta t}+\frac{\mathscr{T}_{j+1}-\mathscr{T}_{j}}{\delta x_{j+\frac{1}{2}}}+\frac{\bar{u}_{j+1}\{\Pi\}_{j+1}-\bar{u}_{j}\{\Pi\}_{j}}{\delta x_{j+\frac{1}{2}}}=0
$$

and

$$
\frac{\bar{\rho}_{j} \bar{E}_{j}-\rho_{j} E_{j}}{\delta t}+\frac{\mathscr{T}_{j+\frac{1}{2}}^{*}-\mathscr{T}_{j-\frac{1}{2}}^{*}}{\delta x_{j}}+\frac{\Pi_{j+\frac{1}{2}}\{\bar{u}\}_{j+\frac{1}{2}}-\Pi_{j-\frac{1}{2}}\{\bar{u}\}_{j-\frac{1}{2}}}{\delta x_{j}}=0 .
$$

\section{Numerical simulations of Riemann problems.}

We perform the numerical resolutions of some Riemann problems - see [4] - on the computational domain $[0,1]$. The number of grid points is equal to 1000 and the time step is given by $\delta t=\delta x / 100$. We take $\gamma=1$.4. The initial data $\rho, u, p$ are piecewise constant functions with a discontinuity located at $x_{0}=0.5$, according to the table below. In Figure 1, we represent the pressure $p_{j+\frac{1}{2}}$, velocity $u_{j}$ and internal energy $e_{j+\frac{1}{2}}$ at the final time $T$ (also given in the table below). 


\begin{tabular}{llllllll}
\hline & $\rho_{l}$ & $\rho_{r}$ & $u_{l}$ & $u_{r}$ & $p_{l}$ & $p_{r}$ & $T$ \\
\hline Test \#1 & 1 & 0.125 & 0 & 0 & 1 & 0.1 & 0.25 \\
Test \#2 & 1 & 1 & 0 & 0 & 1000 & 0.01 & 0.012 \\
Test \#3 & 5.99924 & 5.99242 & 19.5975 & -6.19633 & 460.894 & 46.0950 & 0.035 \\
\hline
\end{tabular}

Test \#1, the so-called Sod test problem, is a mild test whose solution consists of a left rarefaction, a contact disconinuity and a right shock. Test \#2 is a more severe test problem whose solution contains a left rarefaction, a contact discontinuity and a right shock. Test \#3 corresponds to the collision of two strong shocks and consists of a left facing shock (travelling very slowly to the right), a right travelling contact discontinuity and a right travelling shock wave.

Test \#1

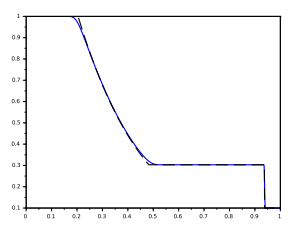

Test \#2

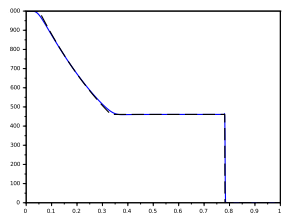

Test \#3

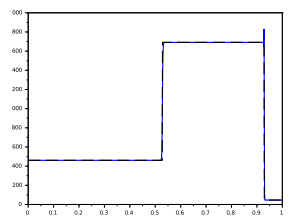

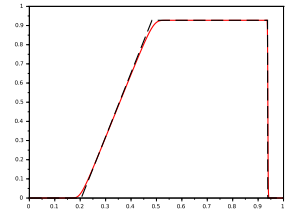
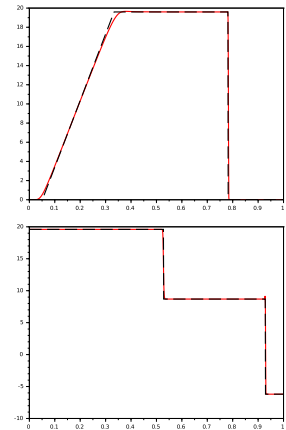
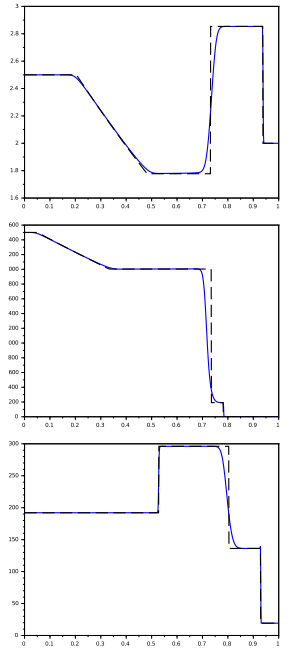

Fig. 1 Numerical (solid lines) and exact (dotted lines) solutions: pressure (left), velocity (middle), internal energy (right).

\section{References}

1. Berthelin, F., Goudon, T., Minjeaud, S.: Kinetic schemes on staggered grids for barotropic Euler models: entropy-stability analysis. Math. Comput. 84, 2221-2262 (2015)

2. Herbin, R., Kheriji, W., Latché, J.C.: Staggered schemes for all speed flows. ESAIM: Proceedings. 35, 122-150 (2012)

3. Herbin, R., Latché, J.C., Nguyen, T.: Consistent explicit staggered schemes for compressible flows. Part II: The Euler equation. hal-00821069 (2013)

4. Toro, E.F.: Riemann solvers and numerical methods for fluid dynamics, third edn. SpringerVerlag, Berlin (2009) 